\section{PMO-031 AN AUDIT INVESTIGATING THE EFFICACY OF THE LOW FODMAP DIET IN IMPROVING SYMPTOMS IN PATIENTS WITH FUNCTIONAL GASTRO-INTESTINAL SYMPTOMS}

doi:10.1136/gutjnl-2012-302514b.31

$\mathrm{J}$ Zubek, ${ }^{*} \mathrm{R}$ White. Department of Nutrition and Dietetics, Barts and the London NHS Trust, London, UK

Introduction The low FODMAP (Fermentable Oligo-, Di-, Monosaccharides and Polyols) diet is a diet low in fermentable carbohydrates. These types of carbohydrate are rapidly fermented but poorly absorbed in the small intestine leading to functional gastrointestinal (GI) symptoms in sensitive individuals. Evidence suggests that a reduction in high FODMAP foods leads to an improvement in GI symptoms. ${ }^{1}$ The aim of this audit was to determine whether the low FODMAP diet was effective in improving symptoms within the first 4-12 weeks of following the diet.

Methods All patients commenced on the low FODMAP diet between July 2010 and June 2011 were included $(n=40)$. Of these 19 patients did not attend their first review and therefore their data were incomplete and they were excluded from the analysis. Patients were asked to score their symptoms subjectively using a symptom severity scale between 0 and $10(0=$ no symptoms/absent; $10=$ severe symptom affecting daily life) at their initial assessment and at subsequent follow-up appointments. Paired sample t-tests were used to compare the difference in symptom scores between the initial appointment and first review. Data were included and results obtained for all patients followed up within 3 months where a score was provided.

Results A statistically significant reduction was seen for bloating, abdominal pain and diarrhoea only.

Conclusion Functional GI symptoms are common and often have a significant impact on a person's quality of life. ${ }^{12}$ These results show that symptoms improved significantly on the low FODMAP diet which is supported by previous studies. ${ }^{1}$ The data presented only measures the response to a low FODMAP diet over a 4-12 week period so we are unable to determine if the improvements seen were maintained in the longer term, where issues such as compliance may have a significant impact. We need to continue to collect data so that we can measure the benefits at 6 and 12 months and investigate compliance over this longer period. In conclusion the low FODMAP diet was shown to be effective in the management and overall improvement of functional GI symptoms, however further evaluation is needed to determine the long term management and effectiveness of this diet.

\section{Abstract PM0-031 Table 1}

\begin{tabular}{lllllllll}
\hline & \multicolumn{3}{l}{ Initial assessment } & & & \multicolumn{2}{l}{ 1st review } & \\
\cline { 2 - 3 } Symptom & $\mathbf{n}$ & Mean & SD & & $\mathbf{n}$ & Mean & SD & p Value \\
\hline Bloating & 9 & 7.33 & 1.35 & & 9 & 5.17 & 2.24 & 0.036 \\
Abdominal pain & 6 & 7.92 & 1.16 & & 6 & 6.00 & 2.10 & 0.020 \\
Diarrhoea & 8 & 7.94 & 1.43 & & 8 & 4.75 & 2.67 & 0.017 \\
Constipation & 3 & 9.17 & 1.04 & & 3 & 9.50 & 0.50 & 0.423 \\
Nausea & 4 & 7.13 & 1.44 & & 6.00 & 2.94 & 0.417
\end{tabular}

${ }^{*}$ Statistically significant, $p$.

Competing interests None declared

\section{REFERENCES}

Gibson P. Shepherd S. J Gastroenterol Hepatol 2009;25:252-8.

2. Barrett J, Gearry R, et al. Dietary poorly absorbed, short chain cabohydrates increase delivery of water and fermentable substrates to the proximal colon. Aliment Pharmacol Ther 2010;31:874-82.

\section{PMO-032 VALIDATION OF A FOOD FREQUENCY QUESTIONNAIRE FOR ESTIMATING CALCIUM INTAKE IN YOUNG FEMALE ADULTS}

doi:10.1136/gutjnl-2012-302514b.32

R Cawley, M Mackenzie-Fraser. ${ }^{*}$ Robert Gordon University, Aberdeen, UK

Introduction The relatively low cost and low respondent burden of a Food Frequency Questionnaire (FFO) that effectively and rapidly identifies individuals with low calcium intakes would be a valuable asset to clinical dietetics. Young female adults (age 18-30 years) tend to have a lower calcium intake which may increase their risk of osteoporosis in later life. This study aimed to examine the validity and reliability of a non-quantitative calcium FFQ by comparing the mean calcium intake measured by the FFQ with that measured by a 7 day weighed food diary.

Methods The validity and reliability of non-quantitative calcium FFO was evaluated. A self-administered FFO was administered to 41 Caucasian female participants, aged $18-26$ years, studying at the Robert Gordon University, Aberdeen. A points system was used to assess whether the individual was meeting their recommended calcium requirement. A paired $t$ test measured the difference between the mean calcium intake measured by the food diary and the FFO.

Results The mean calcium intake estimated by the FFQ and the 7 day weighed food diary was $622.0 \pm 138.3 \mathrm{mg} /$ day and $692.5 \pm 188.1 \mathrm{mg} /$ day, respectively. A paired $t$ test revealed a significant difference of $70.6 \pm 78.1 \mathrm{mg} /$ day between the calcium intake measured by the FFQ and the food diary $(P r=0.931, p<0.05)$

Conclusion A small but significant difference of $70 \mathrm{mg} /$ day was found between the calcium intake measured by the FFO and the 7-day food diary. However, this significant result did not reflect the precision between the two methods at estimating calcium intake. Both Pearson's correlation coefficient and Bland Altman procedures demonstrated good agreement and validity. The FFO identified individuals with low calcium intakes; however, the degree of underestimation by the FFQ increased when calcium intake increased. Although the FFO may not be as accurate as a 7-day weighed food diary when estimating absolute calcium intake it appears to be an effective method of rapidly identifying individuals with low calcium intake. This easily utilised calcium FFQ could be a valuable and useful clinical tool in identifying individuals with calcium deficient diets. Furthur research into whether this FFO is a reliable method of identifying low calcium intakes in other subgroups of the population is recommended.

Competing interests None declared.

\section{REFERENCES}

1. Bates B, Lennox A, Swan G. National diet and nutrition survey. London: Department of Health, 2009

2. Salvini S, et al. Food-based validation of a dietary questionnaire: the effects of weekto-week variation in food consumption. Int J Epidemiol 1989;18:858-67.

3. Tefft M, Boniface D. Estimating food and nutrient intake from food frequency questionnaire data by reference to a standard weighed diet survey. $J$ Hum Nutr Diet 2000;13:219-24.

\section{PM0-033 GLUCOSE AND LIPID REGULATION IS MODULATED BY VASCULAR ADHESION PROTEIN-1 (VAP1) IN NON-ALCOHOLIC FATTY LIVER DISEASE (NAFLD)}

doi:10.1136/gutjnl-2012-302514b.33

${ }^{1} \mathrm{~S}$ Karim, ${ }^{*}{ }^{1} \mathrm{E}$ Liaskou, ${ }^{2} \mathrm{~F}$ Ling, ${ }^{3} \mathrm{~K}$ MacAulay, ${ }^{4} \mathrm{~S}$ Jalkanen, ${ }^{1} \mathrm{D} \mathrm{H}$ Adams, ${ }^{1} \mathrm{P} \mathrm{F}$ Lalor ${ }^{1}$ Immunity \& Infection, University of Birmingham, Birmingham, UK; ${ }^{2} \mathrm{Cell} \&$ Molecular Biology \& Genomics, Unilever R\&D, UK; ${ }^{3}$ Cell \& Molecular Biology \& Genomics, 